\title{
Size-dependent energy levels of CdTe quantum dots
}

\author{
Yasuaki Masumoto and Koji Sonobe* \\ Institute of Physics and Venture Business Laboratory, University of Tsukuba, Tsukuba 305, Japan
}

(Received 17 April 1997)

\begin{abstract}
Successful observation of persistent spectral hole burning in CdTe quantum dots embedded in the glass enabled us to investigate their size-dependent electronic energy levels. Luminescence excitation spectroscopy was utilized to confirm them. The observed size-dependent electronic transitions show a monotonic increase with decrease of the radius from 4.3 to $2.5 \mathrm{~nm}$ and valence level crossing was not observed up to the sixth transition. These experimental results are discussed with reference to the calculated results on the multivalence-band envelope formalism. [S0163-1829(97)02739-2]
\end{abstract}

Quantum size effect of semiconductor nanometer size crystals (nanocrystals) has been one of the targets of extensive research of the optical spectroscopy of semiconductors. Single nanocrystals are ideal for the study of their quantum size effect. Another alternative approach is the site-selective laser spectroscopy of size-dispersed quantum dots. In fact, hole burning and fluorescence line-narrowing spectroscopy are effective to extract the optical properties of single quantum dots from the inhomogeneously broadened optical spectra of the assembly of size-dispersed quantum dots. ${ }^{1,2}$

Recently, persistent spectral hole burning (PSHB) phenomena were observed in many semiconductor quantum dots, such as $\mathrm{CdS}, \mathrm{CdSe}, \mathrm{CuCl}, \mathrm{CuBr}$, and $\mathrm{CuI}$ quantum dots embedded in glass, crystals, or polymers. ${ }^{3}$ These phenomena enable us to investigate precisely the size-dependent energy levels in "laser-marked" quantum dots by observing the site-selectively burned energies in the inhomogeneously broadened absorption spectra. As a result of the persistent spectral hole burning, the electronic and excitonic quantum states including the excited states are burned. This allows us to investigate the quantized energies of quantum dots systematically.

Although the single-band effective-mass model including Coulomb interaction between an electron and a hole gives a successful description of the lowest quantized levels in many semiconductor quantum dots, ${ }^{4,5}$ it does not always apply to the excited quantized levels. Especially, valence-band degeneracy complicates the excited quantized levels in many zincblende semiconductors. ${ }^{6-8}$ In zinc-blende CdTe, the conduction band is made of the $s$ orbital of $\mathrm{Cd}$ and its valence band is made of the $p$ orbital of Te as the first approximation. Orbital angular momentum is mixed with spin angular momentum, and the valence band is split into the topmost $J=$ $\frac{3}{2}$ band and the split-off $J=\frac{1}{2}$ band. Quantum confinement of holes gives an additional angular momentum $L$ for the envelope function, so that the $L-J$ coupling is considered to make complicated energy levels for the hole band. However, CdTe has the largest spin-orbit splitting of $0.927 \mathrm{eV}$ and the smallest band-gap energy, $E_{g}=1.606 \mathrm{eV}$, among $\mathrm{CdS}, \mathrm{CdSe}$, and CdTe. ${ }^{9}$ As a result, the split-off band is expected to mix weakly with the topmost valence band. Additionally, the valence band of zinc-blende CdTe is less complicated than that of wurtzite $\mathrm{CdS}$ and CdSe.
In this paper, PSHB is reported for CdTe quantum dots in the strong confinement regime and its spectroscopic application to the observation of the excited electronic quantum states is carried out. ${ }^{10}$ This spectroscopic tool is compared with luminescence excitation spectroscopy and its utility is evaluated. Size-dependent quantized electronic levels are observed to shift monotonously without any crossing or anticrossing, reflecting the rather simple valence-band structure of CdTe.

Samples studied in this work were CdTe nanocrystals embedded in $\mathrm{GeO}_{2}: \mathrm{Na}_{2} \mathrm{O}$ glass. Molar concentration of 1.5$4.5 \% \mathrm{CdTe}$ mixed with $\mathrm{GeO}_{2}: \mathrm{Na}_{2} \mathrm{O}$ glass was sealed in a quartz ample, melted in a rotating electric furnace at $1200{ }^{\circ} \mathrm{C}$, and quenched. Then, glass pieces were heated for the growth of CdTe nanocrystals. The size of the nanocrystals was controlled by the CdTe doping concentration and the annealing time and was evaluated by means of the smallangle x-ray scattering. It ranges from 2.5 to $5.1 \mathrm{~nm}$.

Samples were directly immersed in superfluid helium at 2 $\mathrm{K}$ for the optical measurement. The excitation light source for the persistent spectral hole burning was a narrow-band dye laser pumped by the second harmonic of the output of a $10 \mathrm{~Hz} Q$-switched $\mathrm{Nd}^{3+}$ :YAG (yttrium aluminum garnet) laser. The spectral linewidth of the dye laser light was 0.014 meV. The absorption spectra of samples were measured by means of a diode-array optical multichannel analyzer or a liquid-nitrogen-cooled charge-coupled-device optical multichannel analyzer equipped with a $25-\mathrm{cm}$ monochromator. The spectral resolution was set to be $1.2 \mathrm{~nm}$. The probe light source was a halogen lamp. Before and after the samples were exposed to the dye laser light pulses, the absorption spectra were measured and the absorption change spectra were derived by subtracting the absorption spectrum taken after the dye laser exposure from that taken before the dye laser exposure.

The luminescence excitation spectra of samples were measured by monitoring the luminescence intensity at the high-energy part of the luminescence band for the better resolution of the excited states. The excitation light source was the $25-\mathrm{cm}$ monochromator output of a $500-\mathrm{W} \mathrm{Xe}$ arc lamp. Its bandwidth was set to be $3 \mathrm{~nm}$. The luminescence was measured by means of a $50-\mathrm{cm}$ monochromator and a photomultiplier. The overall spectral resolution of the excitation spectra was $4.2 \mathrm{~nm}$. 


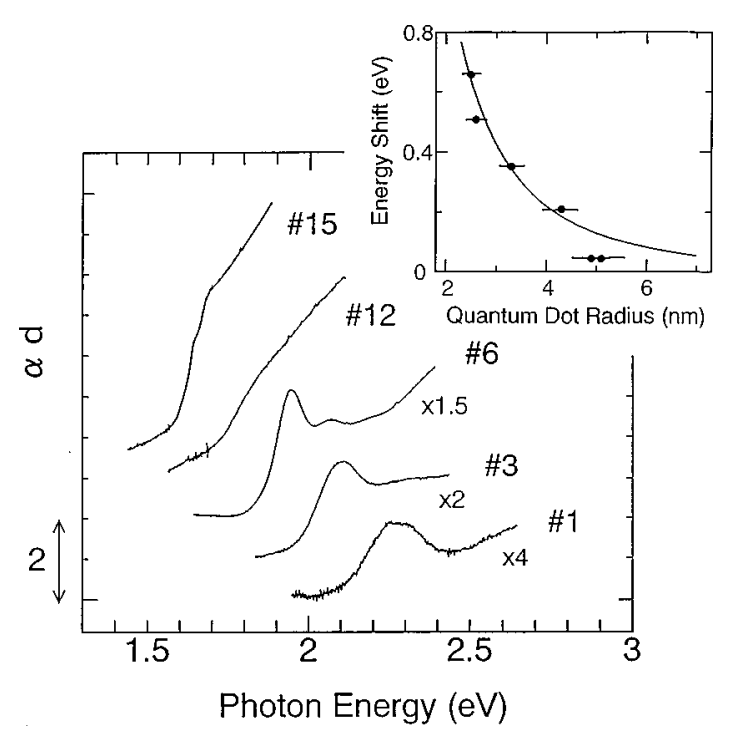

FIG. 1. Optical absorption spectra of five samples, CdTe quantum dots embedded in $\mathrm{GeO}_{2}$ glass, at $2 \mathrm{~K}$. The average radii of dots in samples $1,3,6,12$, and 15 are $2.5,2.6,3.3,4.3$, and $5.1 \mathrm{~nm}$, respectively. In the inset, blueshift of the lowest structures in the absorption spectra from the lowest exciton energy in bulk CdTe, $1.596 \mathrm{eV}$, is shown as a function of the mean radius estimated by the small-angle $x$-ray scattering measurements together with the calculation in the strong confinement regime.

Absorption spectra of five typical samples are shown in Fig. 1. With the decrease of the size, the absorption peak shows blueshift due to the quantum size effect. In the top inset, the blueshift is plotted as a function of the average radius evaluated by means of the small-angle $\mathrm{x}$-ray scattering. The observed blueshift agrees with the calculated one based on the strong confinement model of a spherical quantum dot except the largest size data. The calculation is made by the formula ${ }^{11}$

$$
\Delta E=E-E_{g}+\mathcal{R}=\frac{\hbar^{2} \pi^{2}}{2 \mu R^{2}}-\frac{1.786 e^{2}}{\varepsilon R}+0.752 \mathcal{R},
$$

where $E_{g}=1.606 \mathrm{eV}$ is the band-gap energy, $R$ is the radius of the dot, $\mu=0.0774 m_{0}$ is the reduced mass of an electron mass $m_{e}^{*}=0.096 m_{0}$ and a hole mass $m_{h}^{*}=0.4 m_{0}, \varepsilon=7.1$ is the dielectric constant and $\mathcal{R}=10 \mathrm{meV}$ is the exciton Rydberg energy. ${ }^{9,12}$ Here $m_{0}$ is the electron bare mass. The good agreement between the experimental data and the calculated curve shows that the simple strong confinement model of a spherical quantum dot is enough to explain the blueshift of the lowest quantized level of CdTe quantum dots. Because the largest size is still smaller than twice the exciton Bohr radius, $2 a_{B}=15 \mathrm{~nm},{ }^{13}$ the onset of the intermediate confinement regime, ${ }^{11}$ the disagreement at the largest size regime is not explained by the collapse of the strong confinement model, but probably by the crystal structure transformation to wurtzite structure as discussed later.

Figure 2 shows the inhomogeneously broadened absorption spectra (the upper column) and the absorption spectrum change (the lower column) of the samples, CdTe quantum dots embedded in $\mathrm{GeO}_{2}$ glass. The absorption spectra show a remarkable blueshift with the decrease in the dot size. After the samples are exposed to narrow-band dye laser pulses

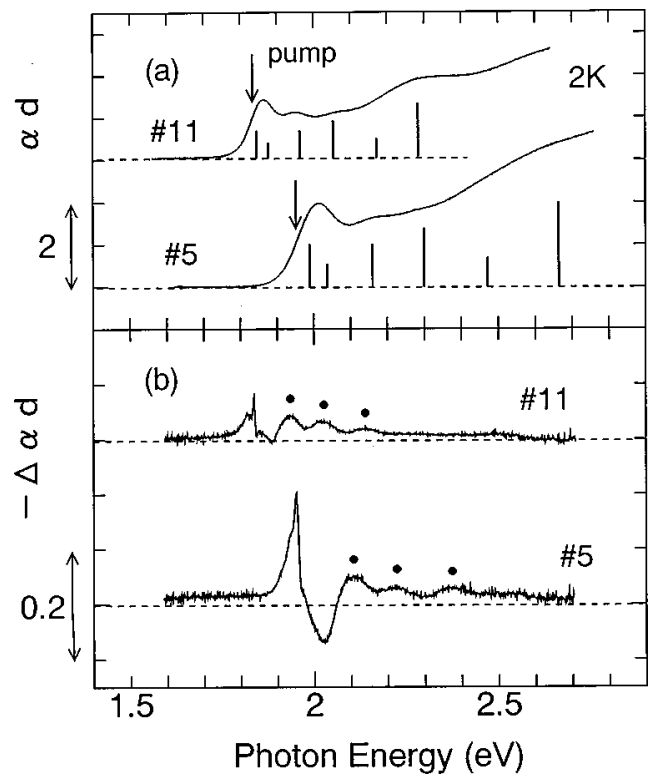

FIG. 2. Optical absorption spectra (upper column) and the absorption change spectra (lower column) of CdTe quantum dots embedded in $\mathrm{GeO}_{2}$ glass at $2 \mathrm{~K}$. The average radii of dots in samples 11 and 5 are 3.7 and $2.9 \mathrm{~nm}$, respectively. Vertical arrows show the burning photon energies of 1.8336 and $1.9526 \mathrm{eV}$, respectively. The absorption change spectra were measured, after the samples were exposed to 1800 shots of dye laser pulses with the excitation energy of $640 \mu \mathrm{J} / \mathrm{cm}^{2}$. Positions of vertical bars in (a) represent the energy positions of the transition deduced from the straight lines in Fig. 4 and their lengths represent the oscillator strength obtained from Ref. 8 , except the $3 S D D_{3 / 2} \rightarrow 1 S_{e}$ transition.

whose photon energy corresponds to the lowest absorption band and after the dye laser exposure was stopped, we started taking the absorption spectrum change at $1.5 \mathrm{~min}$. They showed the hole burning and the burned hole persists for more than $1 \mathrm{~h}$ in contrast with the previous report on the microsecond hole burning of CdTe. ${ }^{13}$ Clear persistent hole burning structures together with the higher-energy satellite structures move with the change of the burning photon energy. The higher-energy satellite structures are considered to be excited states of the burned quantum dots.

It is known that luminescence excitation spectra are useful for the observation of the excited states in quantum dots. ${ }^{14,15}$ Luminescence excitation spectra and PSHB spectra were compared and the validity of the PSHB as a spectroscopic tool was examined. Figure 3 shows the PSHB spectrum and the luminescence excitation spectrum. The higher-energy satellite structures in the absorption spectrum change almost coincide with the structures in the luminescence excitation spectrum. Good coincidence between the PSHB spectrum and the luminescence excitation spectrum indicates the reliability of the peak positions determined by both methods. Although a clear shoulder due to the lowest excited state is observed in the luminescence excitation spectrum, it is not observed at the higher-energy side of the resonantly burned hole in the absorption change spectrum except the absorption increase structure. The absorption increase structure at the higher-energy side of the resonantly burned hole has been often observed in the PSHB spectrum of quantum dots. It seems to mask the excited states around its spectral position 


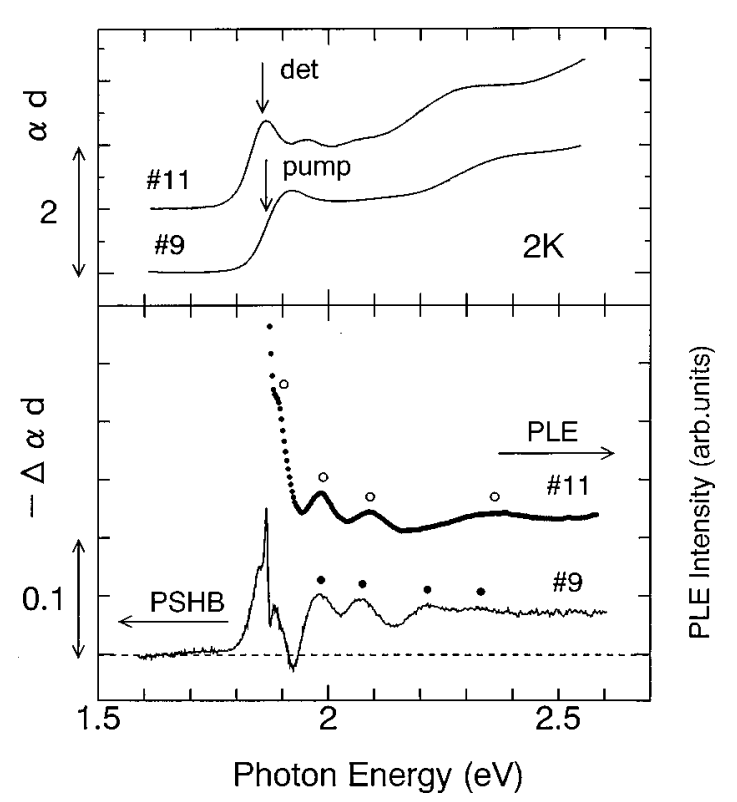

FIG. 3. Comparison of the persistent hole burning spectrum and the luminescence excitation spectrum of CdTe quantum dots embedded in $\mathrm{GeO}_{2}$ glass at $2 \mathrm{~K}$. The average radii of dots in samples 11 and 9 are 3.7 and $3.5 \mathrm{~nm}$, respectively. Downward arrows show the burning photon energy, $1.8678 \mathrm{eV}$, and luminescence detection photon energy, $1.853 \mathrm{eV}$, respectively.

and may give a limitation to the PSHB spectroscopy to investigate the low-energy excited states of quantum dots.

Plotting these transition energies as a function of the excitation photon energy in Fig. 4, we can find straight lines, 1, 2 , and 3 , converging on the bulk-energy fit the experimental data. However, slopes of straight lines 4 and 5 are a little different from those of experimental data. Nevertheless, rather good straight-line fitting meets the two-valence-band theory for CdTe quantum dots where the mixing of the splitoff band is not taken into account. Confinement energies of quantized hole states in CdTe dots were calculated by a multiband effective-mass approximation. By using the calculated results, we can obtain the size-dependent transition energies between quantized hole states and electron states. Therefore, in comparison with the experimental data, we replotted the calculation in which intermixing between three valence bands are considered. ${ }^{8}$ The energy difference between transitions, $2 S D D_{3 / 2} \rightarrow 1 S_{e}, \quad 1 P F P_{3 / 2} \rightarrow 1 P_{e}$, $1 P F F_{5 / 2} \rightarrow 1 P_{e}, 1 P P_{1 / 2} \rightarrow 1 P_{e}$, and $2 P F F_{5 / 2} \rightarrow 1 P_{e}$, and the transition of $1 S D D_{3 / 2} \rightarrow 1 S_{e}$ is plotted by $b, c, d$, e, and $f$, respectively. The comparison shows that the experimental data sets 1,3 , and 4 are identified as transitions $2 S D D_{3 / 2} \rightarrow 1 S_{e}, 1 P F P_{3 / 2} \rightarrow 1 P_{e}$, and $1 P F F_{5 / 2} \rightarrow 1 P_{e}$, respectively. Data set 5 is identified as overlapped transitions, $1 P P_{1 / 2} \rightarrow 1 P_{e}$ and $2 P F F_{5 / 2} \rightarrow 1 P_{e}$. Although experimental data set 2 was not predicted by the theory, they are assigned to be the transition $3 S D D_{3 / 2} \rightarrow 1 S_{e}$. The energy difference between the transition of $3 S D D_{3 / 2} \rightarrow 1 S_{e}$ and the transition of $1 S D D_{3 / 2} \rightarrow 1 S_{e}$ is calculated as is shown by $b^{\prime}$ in Fig. 4 on the basis of the calculated confinement energy of the quantized hole states, $3 S D D_{3 / 2}$ and $1 S D D_{3 / 2} .{ }^{8}$ The calculated energy difference $\Delta E$ between $3 S D D_{3 / 2}$ and $1 S D D_{3 / 2}$ is 3.3 times that between $2 S D D_{3 / 2}$ and $1 S D D_{3 / 2}$. On the other hand, the experimental $\Delta E$ of data set 2 is 3.5 times

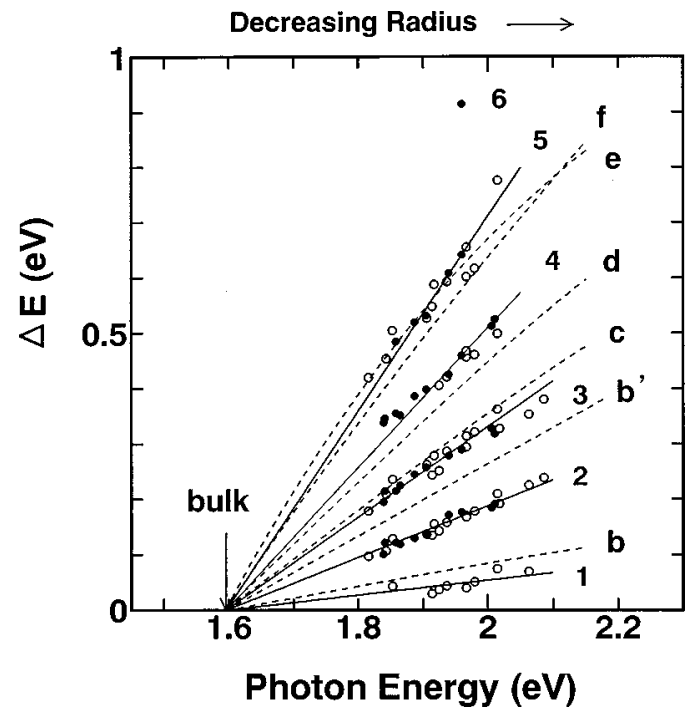

FIG. 4. Excited-state spacings measured relative to the lowest burned structure (center of gravity) shown by solid circles and those measured relative to the luminescence detection energy shown by open circles. Dashed lines labeled by $b, c, d, e$, and $f$ correspond to the transitions $2 S D D_{3 / 2} \rightarrow 1 S_{e}, \quad 1 P F P_{3 / 2} \rightarrow 1 P_{e}$, $1 P F F_{5 / 2} \rightarrow 1 P_{e}, 1 P P_{1 / 2} \rightarrow 1 P_{e}$, and $2 P F F_{5 / 2} \rightarrow 1 P_{e}$ calculated by using the result of Ref. 8 , assuming that the lowest burned structure corresponds to the transition $1 S D D_{3 / 2} \rightarrow 1 S_{e}$. A dashed line labeled by $b^{\prime}$ corresponding to $3 S D D_{3 / 2} \rightarrow 1 S_{e}$ is also calculated by using the result of Ref. 8. Straight lines go through experimental data and a converging point at the lowest exciton transition energy of bulk CdTe, $1.596 \mathrm{eV}$.

$\Delta E$ of data set 1 . If data set 1 is identified as the transition $2 S D D_{3 / 2} \rightarrow 1 S_{e}$, data set 2 is reasonably identified as the transition $3 S D D_{3 / 2} \rightarrow 1 S_{e}$.

The Luttinger parameters $\gamma_{1}$ and $\gamma_{2}$ so far reported or used are scattered, ${ }^{16}$ so that the calculated relative energy positions of the excited states may change crucially depending on the parameter set. Following the previous evaluation of the hole quantized energy of a dot whose radius is $2 \mathrm{~nm}$, the quantized energies of $1 S D D_{3 / 2}$ and $1 P F P_{3 / 2}$ states vary at most by $0.1 \mathrm{eV}$ depending on three sets of Luttinger parameters but the energy separation between $1 S D D_{3 / 2}$ and 1 PFP $_{3 / 2}$ changes at most by $6.4 \mathrm{meV}$. This trial calculation shows that the relative energy between quantized hole states whose principal quantum number for the envelope function is 1 varies little with the change of Luttinger parameters. On the other hand, the energy difference between $1 S D D_{3 / 2}$ and $2 S D D_{3 / 2}$ or $3 S D D_{3 / 2}$ changes by more than $0.1 \mathrm{eV}$. The experimental data are within the estimation uncertainty. Energy splitting of $J=\frac{3}{2}$ hole states depending on the orientation of the crystal can be evaluated by the known $k$-linear term. ${ }^{17}$ The evaluated splitting by the expression $\sqrt{6} K_{l}(\pi / R)$ is $15 \mathrm{meV}$ for $R=2.5 \mathrm{~nm}$, where $K_{l}$ is the coefficient of the $k$-linear term. The value is comparable to data scattering and does not change the relative energy between quantized hole states. It is also noted that the size-dependent Coulomb energy is not taken into account in the calculation. On the other hand, the energy separation between $1 S D D_{3 / 2}$ and $1 P F P_{3 / 2}$ changes considerably, when the effective mass of the electron, $m_{e}^{*}$, varies. It changes from 0.995 to $1.063 \mathrm{eV}$ or 0.957 $\mathrm{eV}$ for a CdTe dot whose radius is $2 \mathrm{~nm}$, when a set of 
parameters, $\gamma_{1}, \gamma_{2}$, and $m_{e}^{*}$, changes from $\gamma_{1}=5.29, \gamma_{2}$ $=1.89$, and $m_{e}^{*}=0.096 m_{0}$ to $\gamma_{1}=5.29, \gamma_{2}=1.89$, and $m_{e}^{*}$ $=0.090 m_{0}$, or $\gamma_{1}=4.7, \gamma_{2}=1.45$, and $m_{e}^{*}=0.099 m_{0}$. The experimental data are within the estimation uncertainty. Therefore, agreement between experimental data and calculation can be improved by the good choice of the parameters.

We also compared our data with that of the previous work on CdTe quantum dots based on the luminescence excitation spectroscopy. ${ }^{18}$ Two data sets of the previous work are very close to lines 1 and 4 in Fig. 4 and a data set lies between lines 2 and 3 . We consider that the second and third excited states are merged and are not resolved well in the previous work.

When the radius of CdTe dots exceeds $4 \mathrm{~nm}$, the absorption spectrum become obscure and almost structureless. Simultaneously, PSHB was not observed. Luminescence excitation spectrum was structureless, too. These observations reflect the missing data region in Fig. 4. The reason why the optical spectra are structureless may be explained by assuming that the samples contain a mixture of CdTe quantum dots of zinc-blende structure and wurtzite structure. ${ }^{19}$ The absorption spectrum of sample 15 whose radius is $5.1 \mathrm{~nm}$ shows two shoulders at 1.6397 and $1.6837 \mathrm{eV}$ as is shown in Fig. 1. Crystal-field splitting is generally observed in wurtzite CdSe and CdS large quantum dots. Energy separation between the two shoulders is $0.044 \mathrm{eV}$, which agrees well with the crystal-field splitting, $0.0465 \mathrm{eV}$, observed in wurtzite bulklike CdTe. The PSHB phenomena should depend strongly on the size of nanocrystals, and should disappear with the increase of the size. This may also explain the presence of the missing data region at the low photon energy regime. Further experimental effort is necessary for the measurement of the size-dependent quantum energy in this large-size region.

In summary, we have successfully observed the PSHB in CdTe quantum dots embedded in $\mathrm{GeO}_{2}: \mathrm{Na}_{2} \mathrm{O}$ glass. The PSHB was utilized to reveal the size-dependent electronic energy levels in CdTe quantum dots as a new site-selective laser spectroscopy. The luminescence excitation spectroscopy was also utilized to investigate them. Good coincidence of the structures revealed by the PSHB and the luminescence excitation spectroscopy show that the PSHB is a reliable spectroscopic tool to investigate the size-dependent electronic energy levels in size-dispersed quantum dots. The observed size-dependent electronic transitions show a monotonic increase with the decrease of the size and valence-band mixing was not present up to the sixth transition. These experimental results are discussed with reference to the calculated results.

The authors wish to thank Dr. S. Nair for valuable discussions and a critical reading of this paper. Small-angle X-ray scattering experiments were done at the Photon Factory (PF) of the National Laboratory for High-Energy Physics by the approval of the PF Advisory Committee (Proposals 95G343). This work was partially supported by a Grand-inAid for Scientific Research No.8454075 from the Ministry of Education, Science, Sports and Culture of Japan.
*Present address: Hino Laboratory, Fuji Electric Corporate Research and Development, Ltd., Asahigaoka, Hino 191, Japan

${ }^{1}$ T. Wamura and Y. Masumoto, Appl. Phys. Lett. 59, 1758 (1991).

${ }^{2}$ T. Itoh and M. Furumiya, J. Lumin. 48\&49, 704 (1991).

${ }^{3}$ For review see Y. Masumoto, J. Lumin. 70, 386 (1996).

${ }^{4}$ A. I. Ekimov, Al. L. Effros, and A. A. Onuschenko, Solid State Commun. 56, 921 (1985).

${ }^{5}$ A. D. Yoffe, Adv. Phys. 42, 173 (1993).

${ }^{6}$ J-B. Xia, Phys. Rev. B 40, 8500 (1989).

${ }^{7}$ A. I. Ekimov, F. Hache, M. C. Schanne-Klein, D. Ricard, and C. Flytzanis, J. Opt. Soc. Am. B 10, 100 (1993).

${ }^{8}$ T. Richard, P. Lefebvre, H. Mathieu, and J. Allègre, Phys. Rev. B 53, 7287 (1996).

${ }^{9}$ Numerical Data and Functional Relationships in Science and Technology, Physics of II-VI and I-VII Compounds, Semimagnetic Semiconductors, edited by O. Madelung, LandoltBörnstein, New Series, Group III, Vol. 17, Pt. b (SpringerVerlag, Berlin, 1982).

${ }^{10}$ Y. Masumoto, K. Sonobe, and N. Sakakura, in Proceedings of the 23rd International Conference on the Physics of Semiconductors, Berlin, 1996, edited by M. Scheffler and R. Zimmermann
(World Scientific, Singapore, 1996), p. 1481.

${ }^{11}$ Y. Kayanuma, Solid State Commun. 59, 405 (1986); Phys. Rev. B 38, 9797 (1988).

${ }^{12}$ Physics and Chemistry of II-VI Compounds, edited by M. Aven and J. S. Prener (North-Holland, Amsterdam, 1967), Chap. 7.

${ }^{13}$ V. Esch, B. Fluegel, G. Khitrova, H. M. Gibbs, Xu. Jiajin, K. Kang, S. W. Koch, L. C. Liu, S. H. Risbud, and N. Peyghambarian, Phys. Rev. B 42, 7450 (1990).

${ }^{14}$ D. J. Norris, A. Sacra, C. B. Murray, and M. G. Bawendi, Phys. Rev. Lett. 72, 2612 (1994).

${ }^{15}$ D. J. Norris and M. G. Bawendi, Phys. Rev. B 53, 16338 (1996).

${ }^{16}$ P. Lefebvre, T. Richard, H. Mathieu, and J. Allègre, Solid State Commun. 98, 303 (1996).

${ }^{17}$ W. Dreybrodt, K. Cho, S. Suga, F. Willmann, and Y. Niji, Phys. Rev. B 21, 4692 (1980).

${ }^{18}$ C. R. M. de Oliveira, A. M. de Paula, F. O. Plentz Filho, J. Medeiros Neto, L. C. Barbosa, O. L. Alves, E. A. Menezes, J. M. M. Rios, H. L. Fragnito, C. H. Brito Cruz, and C. L. Cesar, Appl. Phys. Lett. 66, 439 (1995).

${ }^{19}$ P. Lefebvre, T. Richard, J. Allègre, H. Mathieu, A. CombetteRoos, and W. Granier, Phys. Rev. B 53, 15440 (1996). 